\title{
Nicho ecológico actual y futuro de la Leishmaniasis (Kinetoplastida: Trypanosomatidae) en la región Neotropical
}

\author{
David A. Moo Llanes \\ Centro Regional de Investigación en Salud Pública, Instituto Nacional de Salud Pública, México; \\ davidmooll@gmail.com
}

Recibido 27-VII-2015. Corregido 15-III-2016. Aceptado 04-IV-2016.

\begin{abstract}
Current and future ecological niche of Leishmaniasis (Kinetoplastida: Trypanosomatidae) in the Neotropical region. The leishmaniasis is a complex disease system, caused by the protozoan parasite Leishmania and transmitted to humans by the vector Lutzomyia spp. Since it is listed as a neglected disease according to the World Health Organization, the aim of this study was to determine the current and future niche of cutaneous and visceral leishmaniasis in the Neotropical region. We built the ecological niche model (ENM) of cutaneous ( $\mathrm{N}=2910$ occurrences) and visceral ( $\mathrm{N}=851$ occurrences) leishmaniasis using MaxEnt algorithm. Nine bioclimatic variables (BIO1, BIO4, BIO5, BIO6, BIO7, BIO12, BIO13, BIO14, BIO15 (downloaded from the Worldclim) and disease occurrences data were used for the construction of ENM for three periods (current, 2050 and 2070) and four climate change scenarios (RCP 2.6, 4.5, 6.0 y 8.5). We analyzed the number of pixels occupied, identity niche, modified niche (stable, loss, and gain) and seasonality. Our analyses indicated the expansion for cutaneous leishmaniasis (CL), a comparison for visceral leishmaniasis (VL). We rejected the null hypothesis of niche identity between CL and VL with Hellinger's index $=0.91(0.92-0.98)$ and Schoener's Index $=0.67(0.85-1.00)$ but with an overlap niche of $56.3 \%$. The differences between the two leishmaniasis types were detected in relation to RCP scenarios and niche shifts (area gained / loss). Seasonality was more important for CL. We provided a current picture of CL and VL distributions and the predicted distributional changes associated to different climate change scenarios for the Neotropical region. We can anticipate that increasing range is likely although it will depend locally on the future trends in weather seasonality. Rev. Biol. Trop. 64 (3): 1237-1245. Epub 2016 September 01.
\end{abstract}

Key words: neotropical, leishmaniasis, ecological niche, climate change, seasonal.

La leishmaniasis es una de las principales enfermedades tropicales desatendidas a nivel mundial. Esta enfermedad consiste en cuatro formas clínicas (cutánea localizada, cutánea difusa, mucocutánea y visceral) producidas por diferentes agentes etiológicos, todos ellos parásitos tripanosomatídeos pertenecientes al género Leishmania Ross (Kinetoplastida: Trypanosomatidae), y transmitidas por la picadura de flebotomineos hembras (Psychodidae: Phlebotominae) que afectan a las poblaciones de animales y humanos a lo largo de gran parte de las zonas tropicales y subtropicales. La leishmaniasis se encuentra presente en 98 países a nivel mundial, y es ignorada en las discusiones sobre enfermedades tropicales, ya que apenas en el 2010, se realizó el primer informe técnico después de más de 20 años (WHO, 2010; Alvar et al., 2012).

En América, los ciclos de transmisión de Leishmania implican un gran número de parásitos (14 especies), reservorios, hospederos y vectores. Por lo tanto, dependiendo de la región geográfica, existen un gran número de interacciones entre 62 hospederos mamíferos y 93 especies de flebotomineos, sin mencionar las especies de Leishmania spp. que están potencialmente implicadas en la transmisión (WHO, 2010). En el Nuevo Mundo, la leishmaniasis cutánea (LC) es causada por múltiples especies 
de Leishmania filogenéticamente distintas, presenta distintas formas clínicas, y se encuentra ampliamente distribuida, siendo cinco países (Brasil, Colombia, Perú, Bolivia y Venezuela) los que presentan el mayor número de casos. La leishmaniasis visceral (LV) puede ser endémica o esporádica y el agente etiológico es Leishmania infantum Nicolle, se distribuye en varios países, aunque principalmente en Brasil, Paraguay, Venezuela y Colombia (Alvar et al., 2012). En América, anualmente se reportan aproximadamente 66941 casos de LC y 3 662 casos de LV, sin embargo, no sabemos si estos casos se presentan en localidades únicas (Alvar et al., 2012).

En la actualidad, los modelos de nicho ecológico (MNE) se han usado ampliamente para predecir la distribución geográfica de los vectores y reservorios de la leishmaniasis, tanto en el periodo presente como en escenarios de cambio climático (Peterson \& Shaw, 2003; González et al., 2010; Moo-Llanes et al., 2013). Sin embargo, son escasos los artículos enfocados en el nicho ecológico de Leishmania spp. (King, Campbell-Lendrum, \& Davies, 2004; Nieto, Malone, \& Bavia, 2006; Moo-Llanes et al., 2013; Samy, Campbell, \& Peterson, 2014; Piggot et al., 2014a; Rajabi, Mansourina, Pilesjo, \& Bazmani, 2014); en contraste, con los principales temas que son la distribución espacio-temporal (Feijó et al., 2012; Mollalo, Alimohammandi, Shirzadi, \& Malek, 2015), identificación de áreas de riesgo (Carneiro et al., 2007; Salahi-Moghaddam, Mohebali, Moshfae, Habibi, \& Zarei, 2010; Almeida, de Andrade, \& Werneck, 2011; Seid et al., 2014), determinar la asociación de El Niño con casos de LC y flebotomineos (Cabaniel, Rada, Blanco, Rodríguez-Morales, \& Escalera, 2005; Chaves \& Pascual, 2007; Chaves, Calzada, Valderrama, \& Saldaña, 2014), variabilidad climática de los casos de leishmaniasis (Cárdenas, Sandoval, Rodríguez-Morales, \& Franco-Paredes, 2006) y correlación espacial entre Leishmania spp. y flebotomineos (Chelbi, Kaabi, Bejaoui, Derbali, \& Zhioua, 2009).

Con base en estos argumentos, el objetivo principal de esta investigación fue establecer un panorama del nicho ecológico actual y futuro de la LC y LV en la región Neotropical, que podrían ser de utilidad para evaluar el conocimiento de la distribución de la leishmaniasis, y para predecir el impacto real en la dinámica de transmisión, tanto de los vectores, reservorios o parásitos; y poder establecer estrategias de control en áreas de riesgo de la leishmaniasis, además de medidas de prevención en las nuevas áreas de invasión.

\section{MATERIALES Y MÉTODOS}

Origen de datos: El área de estudio incluye toda la región Neotropical con una extensión de $23676710 \mathrm{~km}^{2}$ en 21 países latinoamericanos y una población estimada de 960 millones de habitantes (www.bankworld.org). Los datos de ocurrencias para casos de leishmaniasis fueron obtenidos de la base global de leishmaniasis de 1960-2012 (Piggot et al., 2014b). La base de datos incluye 2910 registros de localidades únicas de LC y 851 registros de localidades únicas de LV.

Nicho ecológico: Para la construcción de los MNE, se usaron nueve variables bioclimáticas (BIO1, BIO4, BIO5, BIO6, BIO7, BIO12, BIO13, BIO14, BIO15) descargadas de Worldclim (www.worldclim.org/) con un tamaño de pixel de 2.5 minutos de longitud y latitud (aproximadamente $4.5 \mathrm{~km}^{2}$ ). Estas variables fueron seleccionadas de un conjunto de 19 variables, que presentaron una menor inter-correlación $(\mathrm{r}<0.75)$ con un análisis de multicolinealidad (Moo-Llanes et al., 2013). Las variables bioclimáticas y ocurrencias fueron importadas al software gratuito MaxEnt (Phillips, Anderson, \& Schapire, 2006), el cual estima la probabilidad de distribución promedio de encontrar la presencia de probabilidad de máxima entropía, sujeta a una serie de restricciones impuestas por la información disponible sobre la distribución observada de las especies y las condiciones ambientales en el área de estudio. MaxEnt divide el $100 \%$ de los puntos de ocurrencia en datos de entrenamiento para la construcción del modelo $(75 \%)$ y datos de evaluación $(25 \%)$ 
(Anderson, Lew, \& Peterson, 2003). Posteriormente, utilizó los siguientes parámetros: features (linear, product, threshold y hinge), regularización múltiple (2), máximo número de iteraciones (500), y máximo número de background (10 000) y bootstrap (10 réplicas). Después, de obtener el MNE, se realizó la sumatoria de las 10 réplicas para obtener un mapa binario (ausencia / presencia) en base al $95 \%$ de los puntos de ocurrencia. Utilizamos la prueba de "identity test" para evaluar la identidad del nicho ecológico de LC y LV en el periodo actual, mediante los siguientes parámetros: puntos de entrenamiento $(75 \%)$, máximo número de iteraciones (500), tipo de réplica (bootstrap) y threshold (minimum training presence) mediante el software ENMtools (Warren, Glor, \& Turelli, 2010).

Escenarios de cambio climático (ECC): Para los MNE en ECC nos basamos en el $5^{\text {to }}$ Informe de Evaluación (AR5) del Panel Intergubernamental de Cambio Climático (IPCC), en donde se establecen cuatro escenarios de emisiones llamados "Representative Concentration Pathways (RCP)" bajo las categorías de RCP 2.6, 4.5, 6 y 8.5, estos valores representan la estimación de los gases de efecto invernadero (IPCC, 2013). El RCP 2.6 representa una política de mitigación que limita el incremento de la temperatura global a $2{ }^{\circ} \mathrm{C}$, mientras que el RCP 8.5, no incluye una mitigación, resultando en un incremento considerable de los gases de efecto invernadero con una radiación de 8.5 $\mathrm{W} / \mathrm{m}^{2}$ para el año 2100 (Harris et al., 2014). Se seleccionó el modelo de circulación global llamado HadGEM2-AO que incluye "tropósfera, superficie terrestres, hidrología, aerosoles y océano" elaborado por Met Office Hadley Centre (Baek et al., 2013). Las variables bioclimáticas futuras fueron descargadas del Coupled Model Intercomparison Project (www. worldclim.org/cmip5_2.5m), a una resolución de 2.5 minutos para los años 2050 y 2070 .

Los MNE fueron evaluados mediante la prueba de Partial-ROC (Peterson, Papes, \& Soberón, 2008) utilizando el software desarrollado por Barve (2008). Este prueba utilizó los datos de área bajo la curva (AUC), siendo el resultado significativo cuando los valores de AUC son mayores de lo esperado al azar $(\mathrm{AUC}=1.0)$. Se analizó la amplitud del nicho ecológico por periodo de tiempo y ECC, calculando la proporción de pixeles ocupados entre el total del número de pixeles de la región establecida. Se calculó el nicho modificado para el periodo 2050 desde tres perspectivas: hábitat estable (pixeles que se presentan tanto en el periodo actual como en los ECC), hábitat perdido (pixeles que solamente se encontraban en el periodo presente) y hábitat ganado (pixeles que no se encontraban en el periodo presente) (Ramsey et al., 2013; Ikeda, Grady, Shuster, \& Whitham, 2014). Se visualizó el nicho ecológico de LC y LV mediante la gráfica de la $\mathrm{BIO} 1$ versus $\mathrm{BIO} 12$ para el periodo actual, 2050 y 2070 , solamente en el RCP 4.5. Para la prueba de identity test, calculamos los índices Hellinger's y Shoener's para los pares de comparaciones entre LC y LV, versus la distribución nula de lo esperado al azar. La hipótesis de la identidad del nicho se rechaza cuando el valor observado es menor que los valores esperados de las pseudoréplicas (Warren et al., 2010).

\section{RESULTADOS}

Nicho actual: El nicho ecológico de LC, se distribuye desde México hasta Argentina y parte de Chile, a excepción de las regiones elevadas de la Cordillera de los Andes (Fig. 1A). A comparación, del nicho ecológico de LV, que se distribuye en la misma área que la LC pero con algunas áreas de ausencia en México, Colombia, Venezuela, Brasil, Perú, Ecuador, Bolivia y Argentina (Fig. 1B). Ambos modelos de nicho ecológicos fueron considerados significativos, más de lo que se esperaba al azar, con la asociación de los valores de partial-ROC AUC de los modelos mayores a 1. Se rechaza la identity test del nicho ecológico entre LC y LV con el índice Hellinger's $=0.91(0.92-0.98)$ y Schoener's $=0.67$ (0.85-1.00), con un valor de significancia de $\mathrm{P}=0.05$. La contribución de las variables individuales en los nichos ecológicos de LC y LV, resultaron diferentes (Cuadro 1). 

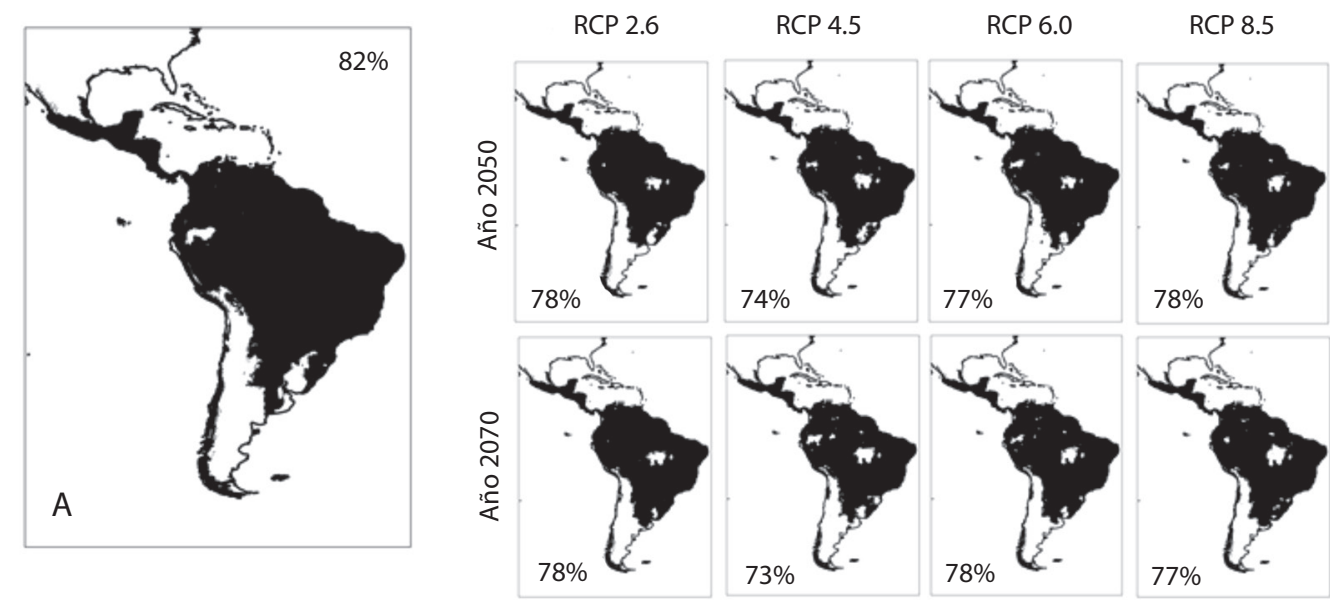

RCP 6.0

RCP 8.5
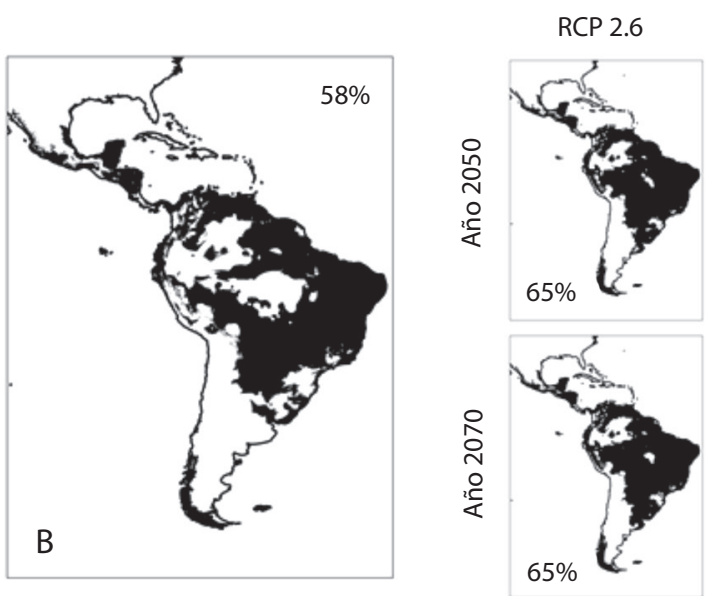

RCP 4.5

RCP 6.0

RCP 8.5
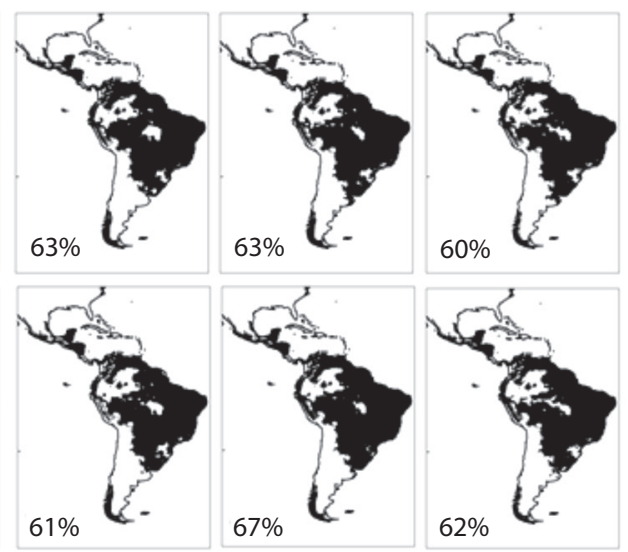

Fig. 1. Modelos de nicho ecológico de leishmaniasis cutánea (A) y visceral (B) en escenarios de cambio climático en la región Neotropical. Los porcentajes corresponden a la cobertura para la región Neotropical.

Fig. 1. Ecological niche models of cutaneous (A) and visceral leishmaniasis (B) in scenarios climate change in the Neotropical region. Percent correspond to the coverage for the Neotropical region.

Nicho futuro: En el periodo actual, se puede observar un traslape del $56.3 \%$ entre el nicho ecológico de LC y LV, presentándose en los países donde ambas se distribuyen, a excepción de Uruguay y Chile, además de un traslape del $55.7 \%$ (año 2050) y $53.5 \%$ (año 2070), estos últimos en el RCP 4.5. La LC presentó una cobertura actual del $82 \%$ en la región Neotropical, con cambios para el 2070 de la siguiente manera: RCP 2.6 (78 \%), RCP 4.5 (73\%), RCP 6 (78 \%) y RCP 8.5 (77\%) (Fig. 1A). Por su parte, la cobertura actual de la LV fue del $58 \%$, RCP 2.6 (65\%), RCP 4.5
(61 \%), RCP 6 (67\%) y RCP 8.5 (62\%) (Fig. 1B). Se visualizó el nicho ecológico, donde LC presenta una estacionalidad en todos los años, a diferencia de LV donde se puede observar una pérdida en los diferentes periodos (Fig. 2).

Nicho modificado: El hábitat estable de la LC en los RCP fue variable desde RCP 2.6 (97.5\%), RCP 4.5 (97.5\%), RCP 6 (97.8\%) y RCP 8.5 (96.7\%). Mientras, que para la LV, la distribución va desde el RCP 2.6 (81.3\%), RCP 4.5 (82.8\%), RCP 6 (82.9\%) hasta el RCP 8.5 $(83.5 \%)$. El hábitat ganado y perdido en la LC 
CUADRO 1

Porcentaje de contribución de las variables bioclimáticas en los modelos de nicho ecológico de leishmaniasis cutánea y leishmaniasis visceral en el periodo actual

TABLE 1

Percent contribution of variables bioclimatic in ecological niche model of cutaneous and visceral leishmaniasis in the current period

\begin{tabular}{cc} 
Leishmaniasis cutánea & Leishmaniasis visceral \\
Bio $5(21.7)$ & Bio $12(34.7)$ \\
Bio $7(19.5)$ & Bio $7(23.3)$ \\
Bio $4(18.3)$ & Bio $1(20.3)$ \\
Bio $13(15.5)$ & Bio 6 $(10.5)$ \\
Bio $15(9.7)$ & Bio $14(3.8)$ \\
Bio $1(7.0)$ & Bio $4(2.4)$ \\
Bio $6(3.9)$ & Bio $5(2.3)$ \\
Bio $14(3.7)$ & Bio $15(1.6)$ \\
Bio 12 $(0.7)$ & Bio $13(1.1)$ \\
\hline
\end{tabular}

fue variable, a diferencia de la $\mathrm{LV}$, donde la ganancia se presenta en un patrón decreciente en los RCP y de una manera inversa para el hábitat perdido (Cuadro 2).

\section{DISCUSIÓN}

La leishmaniasis es una enfermedad tropical prioritaria de la Organización Mundial de la Salud. Esta organización presenta la distribución geográfica de LC y LV para América, teniendo como principal limitante, que no se especifica la metodología para la obtención
CUADRO 2

Porcentaje de pixeles ocupados del nicho modificado de leishmaniasis cutánea y leishmaniasis visceral en 2050

TABLE 2

Percent of occupied pixels of modified niche of cutaneous and visceral leishmaniasis in 2050

\begin{tabular}{lccc}
\multicolumn{1}{c}{ RCP } & Nicho Perdido Nicho Ganado & Nicho Estable \\
& \multicolumn{4}{c}{ Leishmaniasis cutánea } \\
RCP 2.6 & 7.72 & 2.46 & 97.54 \\
RCP 4.5 & 12.58 & 2.29 & 97.59 \\
RCP 6 & 8.89 & 2.16 & 97.80 \\
RCP 8.5 & 8.91 & 3.19 & 96.79 \\
& \multicolumn{4}{c}{} \\
RCP 2.6 & 6.45 & 15.44 & 81.35 \\
RCP 4.5 & 6.47 & 13.93 & 82.35 \\
RCP 6 & 7.00 & 13.77 & 82.90 \\
RCP 8.5 & 7.87 & 12.97 & 83.56 \\
\hline
\end{tabular}

de la distribución. Posteriormente, Piggot et al. (2014a) realizaron un mapa de riesgo de la leishmaniasis global utilizando un árbol de regresión impulsado, con ayuda de cuatro componentes (mapa de la extensión de la enfermedad, mapa de los casos, variables ambientales correlacionadas con la enfermedad y registros de pseudo-datos) con una gran limitante que algunas variables no se pueden proyectar en escenarios futuros. A diferencia de nuestros resultados, en Piggot et al. (2014a) existe área de omisión de LC y LV en la región de Sudamérica, Centroamérica, México y EUA, por

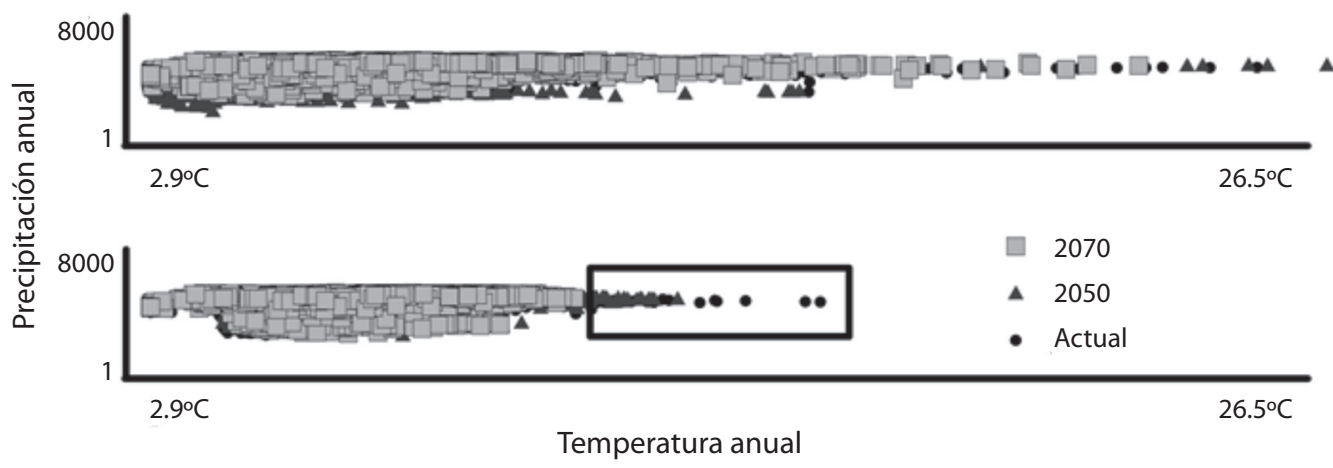

Fig. 2. Visualización del espacio ambiental de leishmaniasis. A) cutánea; B) visceral.

Fig. 2. Viewing the environmental space of leishmaniasis. A) Cutaneous; B) Visceral. 
ejemplo, en estudios focales, la distribución de la leishmaniasis es mayor en el país de interés, tal y como sucede en Colombia (King et al., 2004) y Brasil (Nieto et al., 2006) y México (Moo-Llanes et al., 2013). Es importante destacar, que una de las bondades de nuestros modelos es que son altamente reproducibles a diferentes escalas, se pueden proyectar en escenarios futuros y nos dan un panorama real del nicho ecológico a diferencia de la distribución geográfica actual. Cabe mencionar que la LC y LV, presentan un vacío de información en la región del Amazonas, esto se debe principalmente, al sesgo en la información y en el reporte de los casos de leishmaniasis. Esta enfermedad es multifactorial (CarradaFigueroa, Leal-Ascencio, Jiménez-Sastré, \& López-Álvarez, 2014), por lo tanto, los reportes de casos de leishmaniasis se presentan en las áreas más urbanizadas de las localidades, sin embargo, no hay que menospreciar la exactitud y precisión de nuestros modelos, que pueden darnos un panorama más real del nicho de LC y LV. Es importante, mencionar que no fueron las mismas variables que contribuyeron en los MNE de LC y LV, se puede observar que en la LC sus cambios están influenciados por variables de temperatura mientras que la $\mathrm{LV}$, por variables de precipitación y temperatura. Esto se puede ver reflejado en los resultados de identidad del nicho ecológico, donde ambos (LC y LV) no presentan una similitud entre las condiciones ecológicas.

Es importante señalar, que este es el primer trabajo enfocado al nicho ecológico de la leishmaniasis en la región Neotropical y ECC, aunque, anteriormente se han realizado modelos de nicho ecológico de vectores y reservorios de leishmaniasis en ECC (Peterson \& Shaw, 2003; González et al., 2010; Moo-Llanes et al., 2013). En el 2070, la proporción global de los pixeles ocupados para LC en el Neotrópico es similar en todos los RCP con excepción de RCP 4.5, a diferencia de la LV, donde los RCP 2.6 y RCP 6 presentan el mayor número de pixeles. Otros autores, han evaluado el patrón del incremento de los RCP, por ejemplo, Caminade et al. (2014) encontraron un patrón de incremento en el número de pixeles dependiendo del mayor valor de RCP para la distribución de la Malaria; Saupe, Hendricks, Peterson y Lieberman (2014) encontraron un mayor incremento en el área del número de pixeles para 14 especies de moluscos en el Atlántico Norte. Sin embargo, Shrestha y Bawa (2014) encontraron el mayor incremento en el RCP 2.6 a diferencia de los otros RCP para el hongo Caterpillar (Ophiocordyceps sinensis) en el Himalaya. Cabe mencionar, que Harris et al. (2014) establece una equivalencia entre los escenarios AR5 y el AR4 (IPCC, 2007), el RCP 2.6 no presenta equivalencia en el AR4, el RCP 4.5 es igual al escenario B1, RCP 6 igual al escenario B2 y RCP 8.5 igual al escenario A2. Por lo tanto, en la LC, la amplitud es constante independientemente del RCP, a diferencia de la LV, que el mayor incremento se presenta en el RCP 2.6, considerado un escenario muy improbable por la creación de una medida de mitigación global. Sin embargo, es alarmante saber que también el RCP 6 presentó valores elevados, por la preocupación de la actual tendencia global, por lo que debemos tomar en cuenta este escenario para futura toma de decisiones. Nosotros encontramos un patrón definido para la LV a diferencia de la LC, por ejemplo, García et al. (2014) analizaron 195 especies de anfibios encontrando que se presentan perdida o ganancia en su hábitat adecuado, dependiendo de su vulnerabilidad intrínseca (plasticidad, dispersión y demografía) hacia el cambio climático. Igualmente, Araújo, Thuiller y Pearson (2006) concluyen que hay una pérdida de hábitat para anfibios y reptiles en Europa debido a su escasa capacidad de dispersión. Triviño, Cabeza, Thuiller, Hickler y Araújo (2013) encontraron que las aves ibéricas son negativamente vulnerables al cambio climático modificando su distribución geográfica. Ikeda et al. (2014) encontraron un efecto negativo en las especies de sauces en Norteamérica. Estos autores mencionan un efecto negativo, quizás por la asociación con la temperatura y humedad. Sin embargo, Ramsey et al. (2013) evaluaron el efecto del cambio climático en la enfermedad de Chagas, y encontraron una mayor pérdida 
de hábitat que ganancia, a diferencia de otras enfermedades como la leishmaniasis, malaria y el dengue. Por lo tanto, es importante señalar que existe un incremento global en el número de pixeles por tipo de leishmaniasis. Sin embargo, estos cambios se presentan en diferentes regiones pudiéndose expandir la leishmaniasis a regiones en las que antes no estaba presente. Por otro lado, se encontró que el patrón definido de LC se debe principalmente a la estacionalidad. Estos resultados son similares a los presentados por varios autores, que analizan el impacto de la variabilidad climática (El Niño y la Niña) en un corto periodo de tiempo para la leishmaniasis cutánea en Venezuela (Cabaniel et al., 2005), Colombia (Cárdenas et al., 2006), Costa Rica (Chaves \& Pascual, 2007) y Panamá (Chaves et al., 2014).

En resumen, estos resultados establecen la base para futuros estudios, y el análisis de la estacionalidad y focos locales de la leishmaniasis en los diversos países. Además, ponen de manifiesto el impacto del cambio climático en el nicho ecológico de la leishmaniasis en América, ya que estos resultados podrían servir de guía para futura toma de decisiones.

\section{AGRADECIMIENTOS}

El autor agradece los comentarios de los revisores del manuscrito.

\section{RESUMEN}

La leishmaniasis es un complejo sistema de enfermedades causadas por parásitos protozoarios Leishmania y transmitidos a humanos por el vector Lutzomyia spp, esta es considerada una enfermedad desatendida de acuerdo a la Organización Mundial de la salud. El objetivo de este estudio fue determinar el nicho ecológico actual y futuro de la leishmaniasis cutánea (LC) y leishmaniasis visceral (LV) en la región Neotropical. Nosotros construimos modelos de nicho ecológico (MNE) de LC ( $\mathrm{N}=2910$ ocurrencias) y LV ( $\mathrm{N}=851$ ocurrencias) usando el algoritmo MaxEnt. Nueve variables bioclimáticas (BIO1, BIO4, BIO5, BIO6, BIO7, BIO12, BIO13, BIO14, BIO15; descargadas de Worldclim), y datos de ocurrencias fueron usados para la construcción de los MNE en tres periodos (actual, 2050 y 2070) y cuatro escenarios de cambio climático (RCP 2.6, $4.5,6.0$ y 8.5 ). Nosotros analizamos el número de pixeles ocupados, identidad de nicho, nicho modificado (estable, perdido y ganado) y estacionalidad. Nuestros resultados indican la expansión de la LC en comparación con la LV. Rechazamos la hipótesis nula de identidad de nicho entre LC y LV con un índice de Hellinger's $=0.91$ (0.92-0.98) y Schoener's $=0.67(0.85-1.00)$ pero con un traslape de nicho de $56.3 \%$. El tipo de leishmaniasis tiene diferentes coberturas de acuerdo al RCP y nicho modificado (ganado/ perdido). La LC tiene estacionalidad a diferencia de la LV. Los MNE de LC y LV proporcionar una visión actual de la región Neotropical, que presenta un mayor incremento de acuerdo con el tiempo, sin embargo, el aumento estacional modificaría la invasión de nuevas áreas en la región.

Palabras clave: neotropical, leishmaniasis, nicho ecológico, cambio climático, estacionalidad.

\section{REFERENCIAS}

Almeida, A., de Andrade, R., \& Werneck, G. L. (2011). Identification of risk areas for visceral leishmaniasis in Teresina, Piaui state, Brazil. American Journal of Tropical Medicine and Hygiene, 84(5), 681-687.

Alvar, J., Vélez, I. D., Bern, C., Herrero, M., Desjeux, P., Cano, J., ... the WHO Leishmaniasis Control Team. (2012). Leishmaniasis worldwide and global estimates of its incidence. PLoS ONE, 7(5), e35671.

Anderson, R. P., Lew, D., \& Peterson, A. T. (2003). Evaluating predictive models of species' distributions: criteria for selecting optimal models. Ecological Modelling, 162(3), 211-232.

Araújo, M. B., Thuiller, W., \& Pearson, R. G. (2006). Climate warming and the decline of amphibians and reptiles in Europe. Journal of Biogeography, 3, $1712-1728$

Baek, J. H., Lee, J., Lee, H. S., Hyun, Y. K., Cho, C., Kwon, W. T., ... Byun, Y. H. (2013). Climate change in the 21 st century simulated bye HadGEM-AO under representative concentration pathways. Asia-Pacific Journal of Atmospheric Sciences, 49(5), 603-618.

Barve, N. (2008). Tool for Partial-ROC versión 1. Lawrence, KS, USA: Biodiversity Institute. Retrieved from http://kuscholarworks.ku.edu/dspace/ hadle/1808/10059.2008.

Cabaniel, G., Rada, L., Blanco, J. J., Rodríguez-Morales, A. J., \& Escalera, J. P. (2005). Impacto de los eventos de El Niño Southern Oscillation (ENSO) sobre la leishmaniosis cutánea en Sucre, Venezuela, a través del uso de información satelital, 1994-2003. Revista Peruana de Medicina Experimental de Salud Pública, 22(1), 32-38. Recuperado de www.scielo.org.pe/ pdf/rins/v22n1/a06v22n1.pdf

Caminade, C., Kovats, S., Rocklov, J., Tompkins, A. M., Morse, A. P., Colón-González, F. L., ... Lloyd, S. J. 
(2014). Impact of climate change on global malaria distribution. Proceedings of the National Academy of Sciences of the United States of America, 111(9), 3286-3291.

Cárdenas, R., Sandoval, C. M., Rodríguez-Morales, A. J., \& Franco-Paredes, C. (2006). Impact of climate variability in the occurrence of leishmaniasis in northeastern Colombia. American Journal of Tropical Medicine and Hygiene, 75(2), 273-277. Retrieved from www.ajtmh.org/content/75/2/273.full.pdf + html

Carneiro, D., Bavia, M. E., Rocha, W., Tavares, A., Cardim, L., \& Alemayehu, B. (2007). Application of spatiotemporal scan statistics for the detection of areas with increased risk for American visceral leishmaniasis in the state of Bahia, Brazil. Geospatial Health, 2(1), 113-126.

Carrada-Figueroa, G., Leal-Ascencio, V. J., Jiménez-Sastré, A., \& López-Álvarez, J. (2014). Transmission of cutaneous leishmaniasis associated with cacao (Theobroma cacao) plantations in Tabasco. Gaceta Médica de México, 150, 494-502.

Chaves, L. F., \& Pascual, M. (2007). Climate cycle and forecasts of cutaneous leishmaniasis, a nonstationary vector-borne disease. PLoS Medicine, 4(3), e123.

Chaves, L. F., Calzada, J. E., Valderrama, A., \& Saldaña, A. (2014). Cutaneous leishmaniasis and sand fly fluctuations are associated with El Niño in Panamá. PLoS Neglected Tropical Diseases, 8(10), e3210.

Chelbi, I., Kaabi, B., Bejaoui, M., Derbali, M., \& Zhioua, E. (2009). Spatial correlation between Phlebotomus papatasi Scopoli (Diptera: Psychodidae) and incidence of zoonotic cutaneous leishmaniasis in Tunisia. Journal of Medical Entomology, 46(2), 400-402.

Feijó, V. A., Paes, L. R., Dias, R. A., Amaku, M., Ferreira, J. S., dos Santos, R. B., \& Ferreira, F. (2012). Spacetime cluster analysis of America visceral leishmaniasis in Bauru, Sao Paulo State, Brazil. Caderno do Saúde Pública, 28(10), 1949-1964.

García, R. A., Araújo, M. B., Burgess, N. D., Foden, W. B., Gutsche, A., Rahbek, C., \& Cabeza, M. (2014). Matching species traits to projected threats and opportunities from climate change. Journal of Biogeography, 41, 724-735

González, C., Wang, O., Strutz, S. E., González-Salazar, C., Sánchez-Cordero, V., \& Sarkar, S. (2010). Climate change and risk of leishmaniasis in North America: Predictions from ecological niche models of vector and reservoirs species. PLoS Neglected Tropical Diseases, 4(1), e585.

Harris, R. M., Grose, M. R., Lee, G., Bindoff, N. L., Porfirio, L. L., \& Fox-Hughes, P. (2014). Climate projections for ecologist. WIREs Climate change, $5,621-237$
Ikeda, D. H., Grady, K. C., Shuster, S. M., \& Whitham, T. G. (2014). Incorporating climate change and exotic species into forecasts of Riparian Forest Distribution. PLoS ONE, 9(9), e107037.

IPCC-Intergovernmental Panel on Climate Change (2007). Cambio climático 2007. Informe de sintesis. Contribución de los grupos de trabajo I, II, III al Cuarto Informe de evaluación del Grupo Intergubernamental de Expertos sobre el cambio climático. Ginebra, Suiza.

IPCC-Intergovernmental Panel on Climate Change. (2013). Climate change 2013: The physical science basis. Contributions of Working Group I to the Fifth Assessment Report of the Intergovernmental Panel on Climate Change. United Kingdom and New York: Cambridge University Press.

King, R. J., Campbell-Lendrum, D. H., \& Davies C. R. (2004). Predicting geographic variation in cutaneous leishmaniasis, Colombia. Emerging Infectious Diseases, 10(4), 598-607.

Mollalo, A., Alimohammadi, A., Shirzadi, M. R., \& Malek, M. R. (2015). Geographic information system-based analysis of the spatial and spatio-temporal distribution of zoonotic cutaneous leishmaniasis in Golestan Province, Nort-East of Iran. Zoonoses Public Health, 62(1), 18-28. doi:10.1111/zph.12109

Moo-Llanes, D. A., Ibarra-Cerdeña, C. N., Rebollar-Téllez, E. A., Ibáñez-Bernal, S., González, C., \& Ramsey, J. M. (2013). Current and future niche of North and Central American sand flies (Diptera: Psychodidae) in climate change scenarios. PLoS Neglected Tropical Diseases, 7(9), e2421.

Nieto, P., Malone, J. B., \& Bavia, M. E. (2006). Ecological niche modeling for visceral leishmaniasis in the state of Bahia, Brazil, using genetic algorithm for rule-set prediction and growing degree day-water budget analysis. Geospatial Health, 1(1), 115-126.

Peterson, A. T., \& Shaw, J. J. (2003). Lutzomyia vectors for cutaneous leishmaniasis in Southern Brazil: ecological niche models, predicted geographic distributions, and climate change effects. International Journal of Parasitology, 33(9), 919-931.

Peterson, A. T., Papes, M., \& Soberón, J. (2008). Rethinking receiver operating characteristic analysis applications in ecological niche modeling. Ecological Modelling, 213(1), 63-72.

Phillips, S. J., Anderson, R. P., \& Schapire, R. E. (2006). Maximum entropy modeling of species geographic distributions. Ecological Modelling, 190(3-4), 231-259.

Piggot, D. M., Bhatt, S., Golding, N., Duda, K. A., Battle, K. E., Brady, O. J., ... Hay, S. I. (2014a). Global distributions maps of the leishmaniases. eLife 3, e02851. 
Pigott, D. M., Golding, N., Messina, J. P., Battle, K. E., Duda, K. A., Balard, Y., ... Hay, S. I. (2014b). Global database of leishmaniasis occurrence locations, 19602012. Scientific Data, 1, 140036.

Rajabi, M., Mansourian, A., Pilesjo, P., \& Bazmani, A. (2014). Environmental modelling of visceral leishmaniasis by susceptibility-mapping using neural networks: a case study in north-western Iran. Geospatial Health, 9(1), 179-191.

Ramsey, J. M., Moo-Llanes, D. A., Danis-Lozano, R., Pinto-Castillo, J. F., Ibarra-Cerdeña, C. N., \& CasasMartinez, M. (2013). Peligro de exposición actual y futuro para Dengue, Chagas, leishmaniasis y Paludismo en México. México: CONACyT / SEMARNAT-0108158. Recuperado de http://www.pincc. unam.mx/congresonacional2013/documentos_descargables/PDF/Anfi/RamseyJM\%20.pdf

Salahi-Moghaddam, A., Mohebali, M., Moshfae, A., Habibi, M., \& Zarei, Z. (2010). Ecological study and risk mapping of visceral leishmaniasis in an endemic area of Iran based on a geographical information systems approach. Geospatial Health, 5(1), 71-77.

Samy, A. M., Campbell, L. P., \& Peterson, A. T. (2014). Leishmaniasis transmission: distribution and coarseresolution ecology of two vectors and two parasites in Egypt. Revista da Sociedade Brasileira de Medicina Tropical, 47(1), 57-62.
Saupe, E. E., Hendricks, J. R., Peterson, A. T., \& Lieberman, B. S. (2014). Climate change and marine molluscs of the western North Atlantic: future prospects and perils. Journal of Biogeography, 41, 1352-1366.

Seid, A., Gadisa, E., Tsegaw, T., Abera, A., Teshome, A., Mulugeta, A., Herrero, M., ... Aseffa, A. (2014). Risk map for cutaneous leishmaniasis in Ethiopia based on environmental factors as revealed by geographical information systems and statistics. Geospatial Health, 8(2), 377-387.

Shrestha, U. B., \& Bawa, K. S. (2014). Impact of climate change on potential distribution of Chinese Caterpillar Fungus (Ophiocordyceps sinensis) in Nepal Himalaya. PLoS ONE, 9(9), e106405.

Triviño, M., Cabeza, M., Thuiller, W., Hickler, T., \& Araújo, M. B. (2013). Risk assessment for Iberian birds under global change. Biological Conservation, 168, 192-200.

Warren, D., Glor, R., \& Turelli, M. (2010). ENMtools: a toolbox for comparative studies of environmental niche models. Ecography, 33, 607-611.

WHO-World Health Organization. (2010). Control of the leishmaniasis. Geneva, Switzerland: World Health Organization Technical Report. 
